\title{
Measuring specific attachment relationships of mother, father and peer in Malaysian adolescents
}

\begin{abstract}
The Inventory of Parent and Peer Attachment (IPPA) is the most widely used self-report measure of individual differences in adolescent attachment. However, the factor structure of this measure has not been replicated outside of the Western adolescent population. Using confirmatory factor analysis (CFA), the aim of this study is to explore the factor structure of the IPPA in Malaysian adolescents. A total of 2,040 school-going adolescents across urban and rural areas of Malaysia completed the 75 items of the IPPA Mother, Father and Peer forms. Contrary to Western findings, results revealed that the three factor structure of the original IPPA sets were not replicated in the Malaysian data. A different three-factor structure for the Parental scales and a two-factor structure for the Peer scale were found to best fit the data. Multigroup CFA (MGCFA) of the IPPA-Malay scales supported invariance of the structural model across age, gender and locality of adolescents. The results indicate that assumptions underlying the cross-cultural assessment of attachment relationships need to be examined. Future research is suggested to look into culturally valid instruments to investigate the adolescent attachment relationship beyond the Western context.
\end{abstract}

Keyword: Inventory of parent and peer attachment; Adolescent; Parent; Peer; Attachment 